\title{
Differentiating Spatial Resolution and Detection Limits in Molecular (infrared) Microspectroscopy
}

\author{
Samar Elagamy ${ }^{1}$, Julie Gravelle ${ }^{2}$ and Andre' J. Sommer ${ }^{2}$ \\ 1. Department of Analytical Chemistry, Faculty of Pharmacy, Tanta University, Tanta, Egypt. \\ 2. Molecular Microspectroscopy Laboratory, Miami University, Oxford, USA.
}

Since the revitalization of infrared microspectroscopy in the early 1980's, the method has matured into a routine analytical method employed in a wide variety of scientific disciplines. Like optical microscopy, the spatial resolution of the method is dictated by the Rayleigh criterion. However, unlike optical microscopy, molecular microspectroscopy has an added benefit of increased selectivity. While basic optical microscopy relies on differences in refractive index and morphology to yield contrast, molecular microspectroscopy relies on the selective infrared absorption to provide contrast. Since an infrared spectrum typically contains 3400 individual wavelengths, the selectivity of the method can be significantly enhanced.

The Rayleigh criterion is given by the following equation where " $\lambda$ " is the wavelength of light, "sin $\theta$ " is the half angle acceptance of the objective and " $n$ " is the refractive index of the measurement medium (typically air). Last, "d" is the required separation between two objects in order for them to be resolved.

$$
d=\frac{0.61 \lambda}{n \sin \theta}
$$

The only parameter that can be modified to improve spatial resolution is " $n$ ", since the wavelengths of infrared radiation are fixed between 2.5 and 14 micrometers. In addition, due to manufacturing constraints the typical value for $\sin \theta$ is 0.3 when conducting reflection measurements. Thus the spatial resolution can be maximized by immersing the sample in germanium $(\mathrm{n}=4.0)$ and conducting an attenuated total internal reflection (ATR) measurement [1,2]. Based on a carbonyl absorption of 1700 wavenumbers ( $\sim 5.9$ micrometers) the spatial resolution at this wavelength would be 3 micrometers. One major problem is that new practitioners to the field and those outside the field view this value as an absolute beyond which structures smaller than this dimension cannot be observed. However, this is not the case.

The source for the signal in infrared microspectroscopy is the absorption of light whose magnitude is proportional to the path length of light through the sample and, more importantly, the extinction coefficient of the sample. In an ATR measurement the path length is constant at a given wavelength so the extinction coefficient is most important. Detection of features smaller than the Rayleigh criterion is possible provided the spectral resolution of the measurement is sufficient $[3,4]$. Herein we present an example using polymer laminates in which a layer having dimensions less than the Rayleigh criterion are detected. The model system is a relatively thick polyethylene terephthalate (PET) substrate with a thin layer of polyvinyl acetate (PVA). The laminate was embedded in epoxy resin and cross-sectioned. Figure 1 illustrates an optical image of a cross-section in which the PVA layer measures 1.6 micrometers in thickness. Figure 1 also illustrates the ATR-infrared image and the associated spectra. A principal component regression (PCR) analysis was conducted on the infrared image. The result shows PET as red, PVA as blue and the epoxy as green. The PVA layer is clearly detected. The 
absorption strength (exctinction coefficient) of PVA relative to PET is approximately 1.5. Although images based on the intensity of a single wavenumber, 1733 for PVA and 1716 for PET, clearly show difference in the layers (univariate analysis) PCR analysis uses multiple wavelengths to further enhance those differences.

Figure 2 illustrates an optical image of a cross-section with the PVA layer measuring 0.8 micrometers in thickness. The PVA layer is again clearly detected but is approaching a limit. Detection of still smaller features is dependent on the selectivity of the method and the strength of the extinction coefficients [4].

\section{References:}

[1] Sommer A. J. et al, Appl. Spectrosc. 55 (2001), p. 252.

[2] Gulley-Stahl H. J. et al, Appl. Spectrosc. 64 (2010), p. 15.

[3] Gulley-Stahl H. J. et al, "Evanescent Wave Imaging" in Vibrational Spectroscopic Imaging for Biomedical Applications, ed. G. Srinivasan, (McGraw Hill, New York), p. 99.

[4] Ling C. "Capabilities, Limitations and Applications of ATR-FTIR Imaging" Miami Ph.D. Thesis, (2014), http://rave.ohiolink.edu/etdc/view?acc_num=miamil403627635.
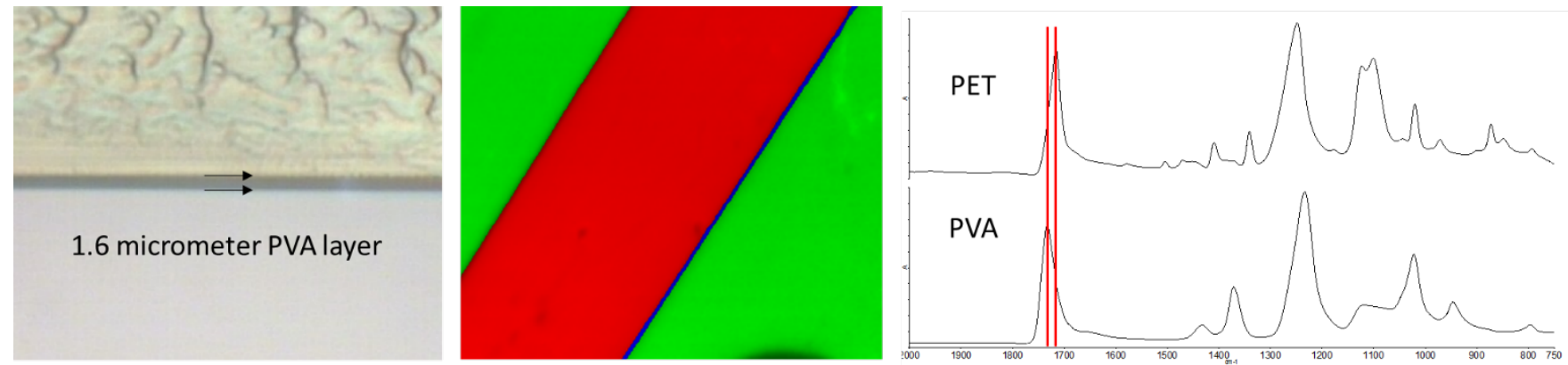

Figure 1. Optical image of the cross-section (left), ATR-infrared image (middle) and spectra of PET and PVA (right).

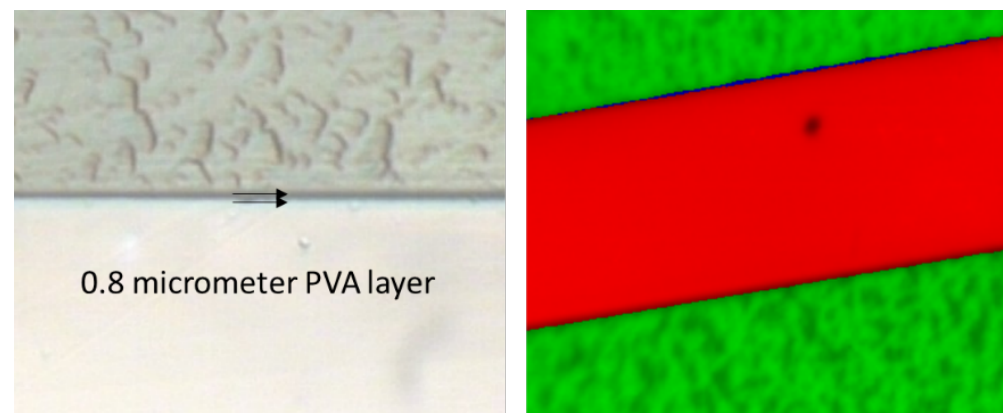

Figure 2. Optical image of the cross-section (left) and ATR-infrared image (right). 